Research Paper

\title{
Effects of Ang II Receptor Blocker Irbesartan on Adipose Tissue Function in Mice with Metabolic Disorders
}

\author{
Akinobu Maeda ${ }^{1}$, Kouichi Tamura ${ }^{\circledR}{ }^{\bowtie}$, Hiromichi Wakui ${ }^{1}$, Masato Ohsawa ${ }^{1}$, Kengo Azushima ${ }^{1}$, Kazushi \\ Uneda ${ }^{1}$, Ryu Kobayashi ${ }^{1}$, Yuko Tsurumi-Ikeya ${ }^{1}$, Tomohiko Kanaoka ${ }^{1}$, Toru Dejima ${ }^{1}$, Koji Ohki ${ }^{1}$, Sona \\ Haku$^{1}$, Akio Yamashita ${ }^{2}$ and Satoshi Umemura ${ }^{1}$ \\ 1. Department of Medical Science and Cardiorenal Medicine, Yokohama City University Graduate Scholl of Medicine, 3-9 Fukuura, Kan- \\ azawa-ku, Yokohama 236-0004, Japan. \\ 2. Department of Molecular Biology, Yokohama City University Graduate Scholl of Medicine, 3-9 Fukuura, Kanazawa-ku, Yokohama \\ 236-0004, Japan.
}

\begin{abstract}
$\square$ Corresponding author: Kouichi Tamura, MD, PhD, Department of Medical Science and Cardiorenal Medicine, Yokohama City University Graduate Scholl of Medicine, 3-9 Fukuura, Kanazawa-ku, Yokohama 236-0004, Japan. Phone: +81 45 787 2635; Fax: +81 45 701 3738; E-mail: tamukou@med.yokohama-cu.ac.jp.

(c) Ivyspring International Publisher. This is an open-access article distributed under the terms of the Creative Commons License (http://creativecommons.org/
\end{abstract} licenses/by-nc-nd/3.0/). Reproduction is permitted for personal, noncommercial use, provided that the article is in whole, unmodified, and properly cited.

Received: 2014.0I.I4; Accepted: 2014.03.3I; Published: 20I4.04.27

\begin{abstract}
Recent studies indicate that the functional renin-angiotensin system (RAS) exists in the adipose tissue. The adipose tissue RAS is proposed in the pathophysiology of metabolic disorders. In the present study, we examined therapeutic effects of irbesartan, an angiotensin II (Ang II) type I receptor (ATIR)-specific blocker, in genetically obese diabetic KKAy mice, a model of human metabolic disorders without any dietary loading, with our focus on the analysis on possible effect of irbesartan on the adipose tissue. The treatment with irbesartan significantly lowered systolic blood pressure with a concomitant decrease in body weight in KKAy mice. In addition, irbesartan significantly decreased the adipose leptin mRNA expression and tended to decrease IL-6 mRNA expression in the adipose tissue of KKAy mice. Furthermore irbesartan preserved the adipose gene expression of ATIR-associated protein (ATRAP), an endogenous inhibitory molecule of tissue ATIR signaling, with a concomitant tendency of up-regulation of adipose tissue ATRAPIATIR ratio. Collectively, these results suggest that the irbesartan-induced beneficial suppressive effect on the leptin-IL-6 axis in the adipose tissue in KKAy mice is partly mediated by a trend of up-regulation of the adipose ATRAPIATIR ratio as one of pleiotropic effects of irbesartan.
\end{abstract}

Key words: adipose tissue; metabolic disorders; inflammation; receptor; renin-angiotensin system.

\section{Introduction}

Metabolic disorders with obesity have become a major medical problem associated with the development of hypertension, type 2 diabetes (T2DM) and dyslipidemia, and ultimately life-threatening cardiovascular and renal diseases. Particularly, adipose tissue has been recently considered as a distinct endocrine organ, which is able to produce adipokines, such as adiponectin and leptin [1]. In addition, dysfunction of adipose tissue is suggested to play a critical role in the development of metabolic disorders through the production of inflammatory cytokines and exaggeration of oxidative stress in the adipose tissue [2].

The renin-angiotensin system (RAS) has been implicated in the pathogenesis of hypertension and cardiovascular disease based on the generation of angiotensin II (Ang II), a key regulator of cardiovas- 
cular homeostasis. Recent evidence has suggested a possible role of RAS in the regulation of adipose tissue function, thereby providing a new perspective concerning the pathophysiology of metabolic disorders and their complications induced by pathological function of tissue RAS [3]. The pathophysiological actions of Ang II are mediated by the Ang II type 1 receptor (AT1R). In the present study, we examined the therapeutic effects of irbesartan, an AT1R-specific blocker, in genetically obese diabetic KKAy mice, a model of human metabolic disorders with T2DM without any dietary loading [4], and focused our analysis on its effect on the adipose tissue.

\section{Materials and Methods}

\section{Animals and Treatment}

Male KKAy mice were purchased from CLEA Japan, Inc. (Tokyo, Japan), and were housed in a controlled environment with a 12-h light-dark cycle and were allowed free access to food and water. Mice were fed a standard diet ( $3.6 \mathrm{kcal} / \mathrm{g}$; $13.3 \%$ energy as fat; Oriental MF, Oriental Yeast, Co., Ltd.). At 9 weeks of age, mice were divided into two groups ( $\mathrm{N}=6-8$ mice per group) for the oral administration of vehicle or irbesartan in drinking water for 4 weeks, and body weight and food intake were measured. The irbesartan dosage $(50 \mathrm{mg} / \mathrm{kg} /$ day) was determined from previous reports using mice [5-7].

Mice were sacrificed under anesthesia and the tissues were collected at the end of the experimental period. The protocol was reviewed and approved by the Animal Studies Committee of Yokohama City University, and all experiments were performed in accordance with the National Institutes of Health guidelines for the use of experimental animals.

\section{Blood Pressure Measurement}

Systolic blood pressure was measured noninvasively by the tail-cuff method (BP-monitor MK-2000; Muromachi Kikai Co.). The MK-2000 BP-monitor made it possible to measure blood pressure without preheating the animals, thus avoiding this very stressful condition [8-10]. At least eight readings were taken for each measurement.

\section{Biochemical Assay}

Blood samples were obtained by cardiac puncture at the time mice were sacrificed in the fed state, unless otherwise stated. Enzymatic assay kits were used for the determination of plasma glucose, free fatty acids, triglycerides and total cholesterol (Wako Pure Chemical, Osaka, Japan). Plasma insulin concentrations were measured with a commercially available ELISA kit (Morinaga, Tokyo, Japan).

\section{Real-time Quantitative Reverse Transcript-PCR (qRT-PCR) Analysis}

Total RNA was extracted from epididymal adipose tissue with ISOGEN (Nippon Gene), and cDNA was synthesized using the SuperScript III First-Strand System (Invitrogen). Real-time quantitative RT-PCR was performed with an ABI PRISM 7000 Sequence Detection System by incubating the reverse transcription product with TaqMan PCR Master Mix and a designed Taqman probe (Applied Biosystems), essentially as described previously [10-14]. The mRNA levels were normalized to those of the $18 \mathrm{~S}$ rRNA control.

\section{Statistical Analysis}

All data are shown as means \pm SEM. Differences were analyzed by Student's unpaired $t$ test. A P-value of $<0.05$ was considered statistically significant.

\section{Results}

\section{Effects of Irbesartan on Blood Pressure, Body Weight, Food Intake and Glucose and Lipid Metabolism}

Systolic blood pressure, heart rate and body weight at baseline in the vehicle-treatment and irbesartan-treatment groups are summarized in Table 1, which shows no significant differences between the groups before the treatment. As shown in Table 2, irbesartan at a dose of $50 \mathrm{mg} / \mathrm{kg}$ per day significantly lowered body weight in KKAy mice with a concomitant decrease in systolic blood pressure. Systolic blood pressure in KKAy mice treated with vehicle was significantly elevated after 4 weeks of the study period compared with baseline (baseline vs. 4 weeks; $107 \pm 2$ vs. $117 \pm 3 \mathrm{mmHg}$, paired $t$-test, $P=0.0109)$. On the other hand, systolic blood pressure in irbesartan-treated KKAy mice did not show evident change after 4 weeks of the study period (baseline vs. 4 weeks; $109 \pm 3$ vs $101 \pm 2 \mathrm{mmHg}$, NS).

Table I. Baseline characteristics of KKAy in the vehicle-treatment and irbesartan-treatment groups.

\begin{tabular}{lccc}
\hline \multicolumn{1}{c}{ Variable } & Vehicle & Irbesartan & $P$ \\
\hline & & & \\
SBP, $\mathrm{mmHg}$ & $107 \pm 2$ & $109 \pm 3$ & 0.5513 \\
HR, bpm & $694 \pm 11$ & $676 \pm 14$ & 0.3304 \\
BW, g & $41.5 \pm 0.3$ & $41.7 \pm 0.2$ & 0.6515
\end{tabular}

The values are mean \pm SEM $(n=6-8)$. Abbreviations: SBP, systolic blood pressure; $\mathrm{HR}$, heart rate; $\mathrm{BW}$, body weight. 
Table 2. Effects of irbesartan on blood pressure, body weight and tissue weight of fat and liver in KKAy mice after 4-week treatment.

\begin{tabular}{lccc}
\hline \multicolumn{1}{c}{ Variable } & Vehicle & Irbesartan & $P$ \\
\hline & & & \\
SBP, mmHg & $117 \pm 3$ & $101 \pm 2^{\star *}$ & 0.0004 \\
HR, bpm & $723 \pm 6$ & $734 \pm 14$ & 0.4301 \\
BW, g & $46.2 \pm 0.3$ & $44.3 \pm 0.9^{\star}$ & 0.0477 \\
Weight of fat tissue, mg & & & \\
$\quad$ Epididymal WAT & $1623 \pm 64$ & $1466 \pm 57$ & 0.1139 \\
$\quad$ Mesenteric WAT & $961 \pm 35$ & $956 \pm 92$ & 0.9558 \\
Fat tissue/BW, \% & & & \\
$\quad$ Epididdymal WAT & $3.7 \pm 0.2$ & $3.4 \pm 0.1$ & 0.2935 \\
$\quad$ Mesenteric WAT & $2.1 \pm 0.1$ & $2.2 \pm 0.2$ & 0.8161 \\
Liver weight, mg & $2936 \pm 104$ & $2875 \pm 247$ & 0.8047 \\
& & & \\
& &
\end{tabular}

The value are mean \pm SEM $(n=6-8)$. Abbreviations: $S B P$, systolic blood pressure; HR, heart rate; $\mathrm{BW}$, body weight, WAT, white adipose tissue. ${ }^{*} \mathrm{P}<0.05,{ }^{* *} \mathrm{P}<0.01 \mathrm{vs}$ vehicle.

On the other hand, heart rate, the wet tissue weight of fat and liver did not significantly differ between the vehicle-treatment and irbesartan-treatment groups. In addition, there was no difference between the two groups in daily food intake (Figure 1). We also examined possible effects of irbesartan on glucose and lipid metabolism in KKAy mice. As shown in Table 3, the irbesartan treatment did not modify the parameters of glucose and lipid metabolism in KKAy mice.

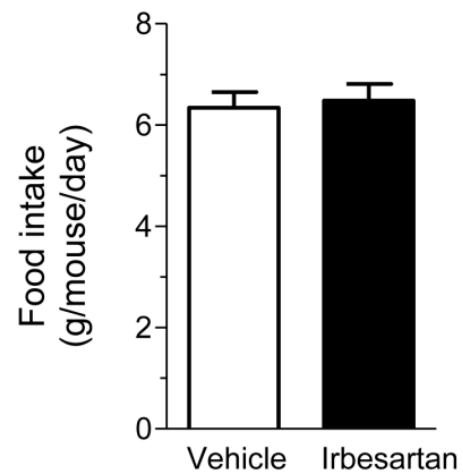

Figure I. Effects of irbesartan on daily food intake in KKAy mice. The values are mean $\pm \operatorname{SEM}(n=8)$.

Table 3. Effects of irbesartan on glucose and lipid metabolism in KKAy mice after 4-week treatment.

\begin{tabular}{llll}
\hline \multicolumn{1}{c}{ Variable } & Vehicle & Irbesartan & $P$ \\
\hline & & & \\
Total cholesterol, $\mathrm{mg} / \mathrm{dl}$ & $154 \pm 5$ & $143 \pm 9$ & 0.3047 \\
Triglyceride, $\mathrm{mg} / \mathrm{dl}$ & $385 \pm 51$ & $450 \pm 113$ & 0.6098 \\
Free fatty acid, mEq/l & $562 \pm 140$ & $619 \pm 83$ & 0.7595 \\
Glucose, $\mathrm{mg} / \mathrm{dl}$ & $524 \pm 33$ & $544 \pm 90$ & 0.8241 \\
Insulin, $\mathrm{ng} / \mathrm{ml}$ & $27.4 \pm 5.0$ & $39.0 \pm 4.9$ & 0.1446
\end{tabular}

The values are mean \pm SEM $(n=6-8)$.
Effects of Irbesartan on Oxidative Stress, Inflammatory Cytokine and Adipokine Gene Expression

We then examined possible effects of irbesartan on expression of the NADPH oxidase components (p22phox, gp91phox) in the epididymal adipose tissue of KKAy mice. As shown in Figure 2, the treatment with irbesartan did not affect the adipose tissue expression of p22phox and gp91phox mRNA. We also analyzed expression of inflammatory cytokine (MCP-1, TNF-alpha, IL-6 and PAI-1) genes in the adipose tissue of KKAy mice and found that the treatment with irbesartan tended to decrease IL-6 mRNA expression $(P=0.099)$ in the adipose tissue of KKAy mice (Figure 3). With respect to a possible effect of irbesartan on adipokines (adiponectin and leptin), while the treatment with irbesartan did not affect the adponectin mRNA expression in the adipose tissue of KKAy mice, irbesartan significantly decreased the adipose leptin mRNA expression $(P=0.023)$ in KKAy mice (Figure 4).

\section{Effects of Irbesartan on Adipose Tissue RAS Gene Expression}

We further examined possible influence of irbesartan on adipose tissue expression of the RAS component genes (angiotensinogen and AT1R) in KKAy mice. While the treatment with irbesartan did not modify the expression of angiotensinogen mRNA in adipose tissue of KKAy mice, irbesartan tended to decrease the adipose AT1R mRNA expression $(P=0.082) \quad$ (Figure 5). Furthermore, the irbesartan treatment preserved the adipose expression of ATRAP mRNA, with a concomitant tendency of up-regulation of adipose tissue ATRAP/AT1R ratio (Figure 5). p22phox

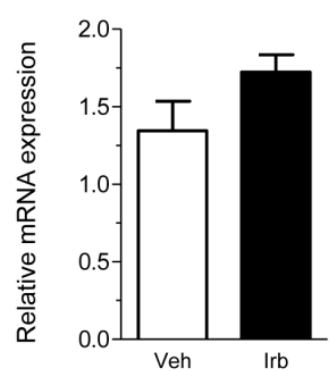

gp91phox

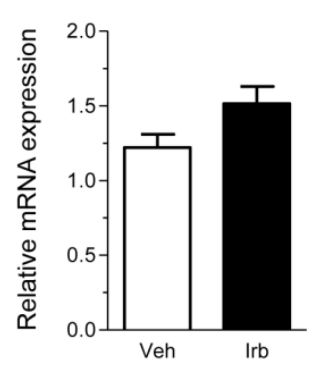

Figure 2. Effects of irbesartan on the adipose tissue mRNA expression of NADPH oxidase components (p22phox and gp9l phox) in KKAy mice. The values are mean $\pm \operatorname{SEM}(n=6-8)$. Veh, vehicle; Irb, irbesartan. 

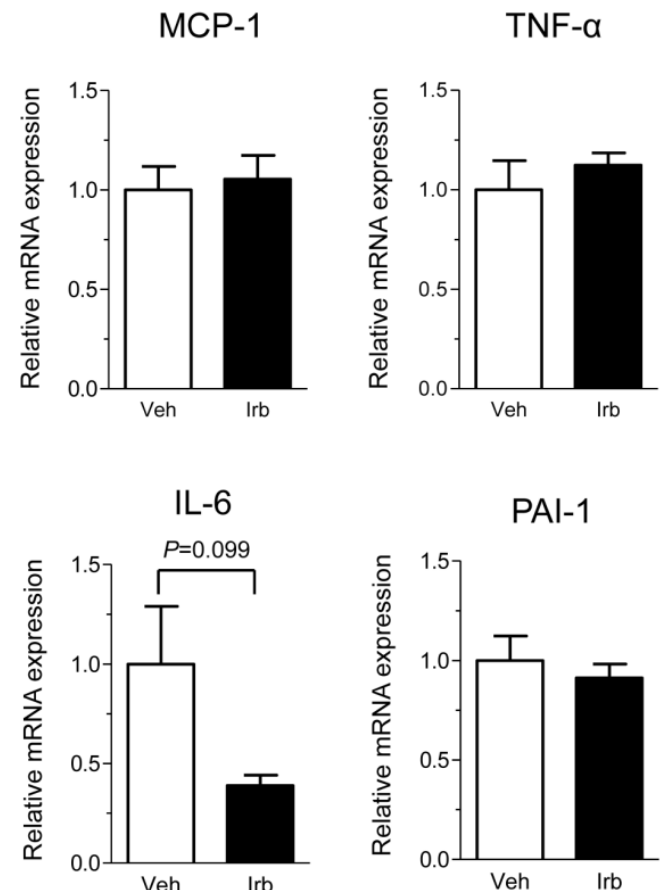

Figure 3. Effects of irbesartan on the adipose tissue mRNA expression of inflammatory cytokines (MCP-I, TNF- $\alpha$, IL-6 and PAI-I) in KKAy mice. The values are mean \pm SEM $(n=6-8)$. Veh, vehicle; Irb, irbesartan.
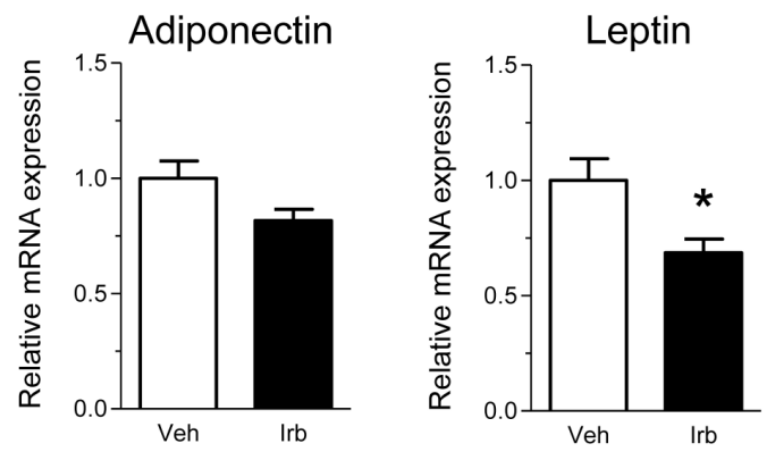

Figure 4. Effects of irbesartan on the adipose tissue mRNA expression of adipokines (adiponectin and leptin) in KKAy mice. The values are mean \pm SEM $(n=6-8)$. Veh, vehicle; Irb, irbesartan. $* P<0.05$ vs vehicle.

\section{Discussion}

Accumulated evidence indicates that adipose tissue is an important source of inflammatory cytokines, adipokines and endocrine factors with broad physiological and pathophysiological effects. Particularly, there is a substantial expression of RAS component genes, such as angiotensinogen and AT1R, in the adipose tissue, and the adipose tissue RAS is proposed to play a role in the regulation of adipocyte function. Furthermore, the pathological activation of adipose tissue RAS is suggested to be involved in the pathophysiology of metabolic disorders via dysregulation of production of oxidative stress, inflammatory cytokines and adipokines in the adipose tissue. Thus, it is important to investigate a possible beneficial ef- fect of therapeutic interventions on adipose tissue function including an influence on the adipose tissue RAS for the development of more efficient strategy to treat metabolic disorders.

In the present study, we showed that the treatment with irbesartan for 4 weeks significantly decreased blood pressure in KKAy mice. In addition, the irbesartan treatment provoked a significant reduction in BW without suppression of dietary food intake. Thus, the hypotensive effect of irbesartan with a concomitant anti-obesity effect may be mediated via pleiotropic effects of irbesartan on the adipose tissue function in addition to its blood pressure lowering effect. From this point of view, there are three interesting findings possibly related to pleiotropic effects of irbesartan on adipose tissue in KKAy mice; 1) an inhibitory effect on IL-6 gene expression, 2) a suppressive effect on leptin gene expression and 3) a tendency of improvement in tissue expression balance between ATRAP and AT1R.
Angiotensinogen
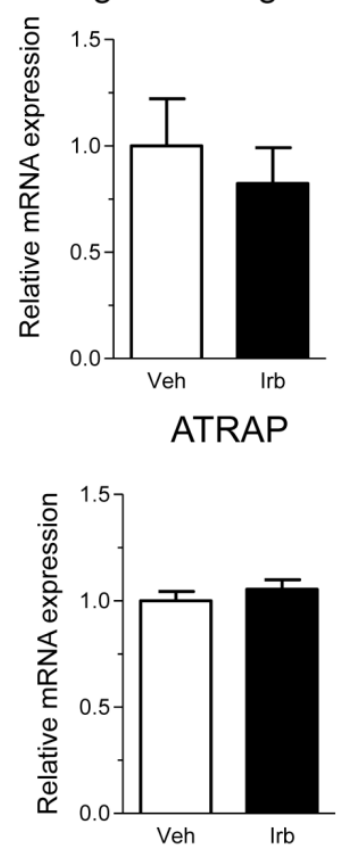

AT1R

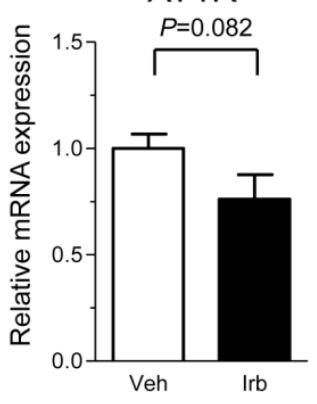

ATRAP/ AT1R

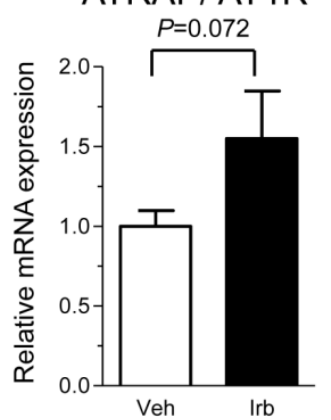

Figure 5. Effects of irbesartan on the adipose tissue mRNA expression of angiotensinogen, ATIR and ATRAP in KKAy mice. The values are mean \pm SEM $(n=6-8)$. Veh, vehicle; Irb, irbesartan.

The chronic low-grade activation of an inflammatory response in adipose tissue is reported to be involved in the pathogenesis of metabolic disorders with obesity [15-17], and the exaggeration of adipose tissue inflammation occurs via the secretion of proinflammatory cytokines and factors derived from adipocytes [18]. In the present study, irbesartan significantly inhibited the adipose tissue gene expression of IL-6, which is one of key players in the inflammatory 
process in adipose tissue in metabolic disorders [17, 19]. Adiponectin and leptin were well-established endocrine factors involved in the pathophysiology of metabolic disorders $[20,21]$.

Although irbesartan reportedly has a stimulatory effect on adiponectin production in cultured adipocytes and in the adipose tissues of obese Zucker rats and apolipoprotein E-deficient mice [7, 22, 23], irbesartan treatment did not affect the adiponectin mRNA expression in the adipose tissue of KKAy mice in the present study. This discrepancy regarding the promoting influence of irbesartan on adiponectin would be due to difference in experimental animal models used for analysis. Alternatively, a post-transcriptional modulation may play a role in the irbesartan-mediated effects on adiponectin production in adipose pathophysiology as suggested in previous studies [24, 25].

Leptin is the firstly characterized adipokine, which is $16 \mathrm{kDa}$ peptide hormone encoded by the $o b$ gene and mainly produced by white adipose tissue regulated by energy level, food intake, several hormones and various inflammatory mediators [26, 27]. Leptin provides the functional link between the immune system and energy homoeostasis [28]. Leptin has been shown to up-regulate various mediators of vascular inflammation including IL-6 [29]. With respect to cardiovascular disease, leptin is suggested to have a variety of pro-atherogenic functions [30]. Thus, through its inflammatory and pro-atherogenic properties, leptin plays an important pro-inflammatory role in metabolic disorders in obesity, thereby providing a functional link between obesity and cardiovascular disease.

In the present study, irbesartan exhibited an inhibitory effect on the leptin-IL-6 axis with anti-obesity effect in KKAy mice, a human model of metabolic disorders. However, It should be determined why the irbesartan treatment exerted a tendency of suppression only on IL-6 mRNA expression among inflammatory cytokines in the adipose tissue of KKAy mice. Although the IL-6-specific post-transcriptional gene regulatory mechanism in the adipose tissue would be a relevant mechanism the selective inhibitory effect of irbesartan on IL-6 expression [31], further investigation is necessary to elucidate the molecular mechanism in detail.

We previously cloned ATRAP as a molecule which interacts with AT1R, and showed that ATRAP suppressed the Ang II-induced hypertrophic and proliferative responses of cardiovascular cells by inducing AT1R internalization [32, 33]. Thus, a tissue-specific regulatory balancing of ATRAP and AT1R expression may be involved in the modulation of AT1R signaling in each tissue [34-37]. We previ- ously showed a tissue-specific regulatory balancing of the expression of ATRAP and AT1R during the development of hypertension and cardiac remodeling in spontaneously hypertensive rats (SHR) and suggested that the up-regulation of cardiac ATRAP/AT1R ratio may be one of the therapeutic benefits [38]. We also found that prepubertal transient blockade of AT1R signaling exerts a long-term therapeutic effect on salt-induced hypertension and renal injury in Dahl Iwai salt-sensitive rats, partly through a sustained enhancement of renal ATRAP expression, thereby suggesting ATRAP a novel molecular target in salt-induced hypertension and renal injury [39]. In the present study, the treatment with irbesartan exerted an inhibitory influence on the adipose tissue AT1R gene expression with a concomitant preservation of adipose tissue ATRAP gene expression, thereby improving the adipose tissue expression balance between ATRAP and AT1R in KKAy mice. Therefore, it is suggested that the irbesartan-induced beneficial suppressive effect on the leptin-IL-6 axis in the adipose tissue in KKAy mice is partly mediated by a trend of up-regulation of the adipose ATRAP/AT1R ratio as one of pleiotropic effects of irbesartan.

\section{Conclusion}

The results of present study showed beneficial therapeutic effects of irbesartan on the adipose tissue in addition to its blood pressure lowering effect in a mouse model of human metabolic disorders. The results also suggest that the irbesartan-induced beneficial suppressive effect on the leptin-IL-6 axis in the adipose tissue in KKAy mice is partly mediated by a trend of up-regulation of the adipose ATRAP/AT1R ratio as one of pleiotropic effects of irbesartan.

\section{Acknowledgements}

Irbesartan was generously gifted from Dainippon Sumitomo Pharma Co., Ltd (Osaka, Japan). This work was supported by a Health and Labor Sciences Research grant and by grants from the Japanese Ministry of Education, Science, Sports and Culture, the Salt Science Research Foundation (No. 1428), the Yokohama Foundation for Advancement of Medical Science and the Kidney Foundation, Japan (JKFB13-17).

\section{Competing Interests}

$\mathrm{K}$. Tamura received honoraria, consulting fees or funds from Novartis, Takeda, Daiichi-Sankyo, Dainippon-Sumitomo, Kyowa-hakko Kirin, Chu-gai, Shionogi, Boehringer Ingelheim, Astellas, Mochida, Pfizer, Mitsubishi Tanabe and Sanofi. 


\section{References}

1. Maury E, Brichard S M. Adipokine dysregulation, adipose tissue inflammation and metabolic syndrome. Mol Cell Endocrinol. 2010; 314: 1-16.

2. Hajer G R, van Haeften T W, Visseren F L. Adipose tissue dysfunction in obesity, diabetes, and vascular diseases. Eur Heart J. 2008; 29: 2959-71.

3. Jing F, Mogi M, Horiuchi M. Role of renin-angiotensin-aldosterone system in adipose tissue dysfunction. Mol Cell Endocrinol. 2012.

4. Suto J, Matsuura S, Imamura K, et al. Genetic analysis of non-insulin-dependent diabetes mellitus in KK and KK-Ay mice. Eur J Endocrinol. 1998; 139: 654-61.

5. Dol F, Martin G, Staels B, et al. Angiotensin AT1 receptor antagonist irbesartan decreases lesion size, chemokine expression, and macrophage accumulation in apolipoprotein E-deficient mice. J Cardiovasc Pharmacol. 2001; 38: 395-405.

6. Liu B C, Xia H L, Wu J N, et al. Influence of irbesartan on expression of ILK and its relationship with epithelial-mesenchymal transition in mice with unilateral ureteral obstruction. Acta Pharmacol Sin. 2007; 28: 1810-8.

7. Iwai M, Kanno H, Senba I, et al. Irbesartan increased PPARgamma activity in vivo in white adipose tissue of atherosclerotic mice and improved adipose tissue dysfunction. Biochem Biophys Res Commun. 2011; 406: 123-6.

8. Iwamoto T, Kita S, Zhang J, et al. Salt-sensitive hypertension is triggered by $\mathrm{Ca} 2+$ entry via $\mathrm{Na}+\mathrm{Ca} 2+$ exchanger type- 1 in vascular smooth muscle. Nat Med. 2004; 10: 1193-9.

9. Tsurumi Y, Tamura K, Tanaka Y, et al. Interacting molecule of AT1 receptor, ATRAP, is colocalized with AT1 receptor in the mouse renal tubules. Kidney Int. 2006; 69: 488-94

10. Wakui H, Tamura K, Matsuda M, et al. Intrarenal suppression of angiotensin II type 1 receptor binding molecule in angiotensin II-infused mice. Am J Physiol Renal Physiol. 2010; 299: F991-F1003.

11. Matsuda M, Tamura K, Wakui $\mathrm{H}$, et al. Upstream stimulatory factors 1 and 2 mediate the transcription of angiotensin II binding and inhibitory protein. J Biol Chem. 2013; 288: 19238-49.

12. Wakui H, Tamura K, Tanaka Y, et al. Cardiac-specific activation of angiotensin II type 1 receptor-associated protein completely suppresses cardiac hypertrophy in chronic angiotensin II-infused mice. Hypertension. 2010; 55: 1157-64.

13. Wakui $\mathrm{H}$, Tamura $\mathrm{K}$, Masuda $\mathrm{S}$ I, et al. Enhanced angiotensin receptor-associated protein in renal tubule suppresses angiotensin-dependent hypertension. Hypertension. 2013; 61: 1203-10.

14. Wakui H, Dejima T, Tamura $K$, et al. Activation of angiotensin II type 1 receptor-associated protein exerts an inhibitory effect on vascular hypertrophy and oxidative stress in angiotensin II-mediated hypertension. Cardiovasc Res. 2013; 100: 511-9.

15. Apovian C M, Bigornia S, Mott M, et al. Adipose macrophage infiltration is associated with insulin resistance and vascular endothelial dysfunction in obese subjects. Arterioscler Thromb Vasc Biol. 2008; 28: 1654-9.

16. Neels J G, Olefsky J M. Inflamed fat: what starts the fire? J Clin Invest 2006; 116: 33-5.

17. Schenk S, Saberi M, Olefsky J M. Insulin sensitivity: modulation by nutrients and inflammation. J Clin Invest 2008; 118: 2992-3002.

18. Tabata M, Kadomatsu T, Fukuhara $S$, et al. Angiopoietin-like protein 2 promotes chronic adipose tissue inflammation and obesity-related systemic insulin resistance. Cell Metab. 2009; 10: 178-88.

19. Lazar M A. The humoral side of insulin resistance. Nat Med. 2006; 12:43-4.

20. Yamauchi T, Kamon J, Waki H, et al. The fat-derived hormone adiponectin reverses insulin resistance associated with both lipoatrophy and obesity. Nat Med. 2001; 7: 941-6.

21. Mattu H S, Randeva H S. Role of adipokines in cardiovascular disease. J Endocrinol. 2013; 216: T17-36

22. Clasen R, Schupp M, Foryst-Ludwig A, et al. PPARgamma-activating angiotensin type-1 receptor blockers induce adiponectin. Hypertension. 2005; 46: 137-43.

23. Fujino M, Miura S, Kiya Y, et al. A small difference in the molecular structure of angiotensin II receptor blockers induces AT(1) receptor-dependent and -independent beneficial effects. Hypertens Res. 2010; 33: 1044-52.

24. Banga A, Unal R, Tripathi P, et al. Adiponectin translation is increased by the PPARgamma agonists pioglitazone and omega-3 fatty acids. Am J Physiol Endocrinol Metab. 2009; 296: E480-9.

25. Liu M, Liu F. Transcriptional and post-translational regulation of adiponectin. Biochem J. 2010; 425: 41-52

26. Matarese G, Mantzoros C, La Cava A. Leptin and adipocytokines: bridging the gap between immunity and atherosclerosis. Curr Pharm Des. 2007; 13: 3676-80.

27. Zhang Y, Proenca R, Maffei M, et al. Positional cloning of the mouse obese gene and its human homologue. Nature. 1994; 372: 425-32.

28. Lago F, Dieguez C, Gomez-Reino J, et al. Adipokines as emerging mediators of immune response and inflammation. Nat Clin Pract Rheumatol. 2007; 3: 716-24.

29. Bouloumie A, Marumo T, Lafontan M, et al. Leptin induces oxidative stress in human endothelial cells. FASEB J. 1999; 13: 1231-8.

30. Beltowski J. Leptin and atherosclerosis. Atherosclerosis. 2006; 189: 47-60.

31. Matsushita K, Takeuchi O, Standley D M, et al. Zc3h12a is an RNase essential for controlling immune responses by regulating mRNA decay. Nature. 2009; 458: $1185-90$.
32. Azuma K, Tamura K, Shigenaga A, et al. Novel regulatory effect of angiotensin II type 1 receptor-interacting molecule on vascular smooth muscle cells. Hypertension. 2007; 50: 926-32.

33. Tanaka Y, Tamura K, Koide Y, et al. The novel angiotensin II type 1 receptor (AT1R)-associated protein ATRAP downregulates AT1R and ameliorates cardiomyocyte hypertrophy. FEBS Lett. 2005; 579: 1579-86.

34. Horiuchi M, Iwanami J, Mogi M. Regulation of angiotensin II receptors beyond the classical pathway. Clin Sci. 2012; 123: 193-203.

35. Mogi M, Iwai M, Horiuchi M. Emerging concepts of regulation of angiotensin II receptors: new players and targets for traditional receptors. Arterioscler Thromb Vasc Biol. 2007; 27: 2532-9.

36. Tamura K, Tanaka Y, Tsurumi $\mathrm{Y}$, et al. The role of angiotensin AT1 receptor-associated protein in renin-angiotensin system regulation and function. Curr Hypertens Rep. 2007; 9: 121-7.

37. Tamura K, Wakui H, Maeda A, et al. The physiology and pathophysiology of a novel angiotensin receptor-binding protein ATRAP/Agtrap. Curr Pharm Des. 2013; 19: 3043-48.

38. Shigenaga A, Tamura $\mathrm{K}$, Wakui $\mathrm{H}$, et al. Effect of olmesartan on tissue expression balance between angiotensin II receptor and its inhibitory binding molecule. Hypertension. 2008; 52: 672-8.

39. Dejima T, Tamura K, Wakui H, et al. Prepubertal angiotensin blockade exerts long-term therapeutic effect through sustained ATRAP activation in salt-sensitive hypertensive rats. J Hypertens. 2011; 29: 1919-29. 